Discrete Comput Geom 26:493-497 (2001)

DOI: $10.1007 / \mathrm{s} 00454-001-0046-7$

\title{
Lines Tangent to Four Unit Spheres with Coplanar Centres
}

\author{
G. Megyesi \\ Department of Mathematics, UMIST, \\ P.O. Box 88, Manchester, M60 1QD, England \\ gmegyesi@am.ma.umist.ac.uk
}

Abstract. We prove that there are at most eight lines tangent to four unit spheres in $\mathbb{R}^{3}$ if the centres of the spheres are coplanar, but not collinear. This bound is sharp.

Macdonald et al. proved in Theorem 1 of [2] that the maximum number of lines tangent to four unit spheres in $\mathbb{R}^{3}$ is twelve, unless the centres of the spheres are collinear. They remark that twelve might not be the best bound if the centres are affinely dependent. We prove that this is indeed the case.

Theorem 1. If the centres of four unit spheres in $\mathbb{R}^{3}$ are coplanar but not collinear, then there are at most eight lines tangent to all four spheres, and this bound is sharp.

Proof. We use the idea from [1] that lines tangent to the four spheres correspond to circular cylinders of radius 1 passing through the four centres. The cylinder intersects the plane of the four centres either in an ellipse with semi-minor axis of length 1 , or in two parallel lines at distance at most 2 from each other, passing through the centres of the four spheres. Each such ellipse or pair of parallel lines corresponds to at most two cylinders, therefore it gives rise to at most two lines tangent to the four spheres.

We prove that given four non-collinear points in $\mathbb{R}^{2}$, there are at most four ellipses with semi-minor axis of length 1 or pairs of parallel lines passing through them.

We consider a possibly degenerate conic $K \subset \mathbb{R}^{2}$ given by the equation

$$
Q(x, y)=a x^{2}+2 h x y+b y^{2}+2 g x+2 f y+d=0,
$$

where $a, b, d, f, g, h \in \mathbb{R}$. We assume that $a, b$ and $h$ are not all 0 .

First, we rotate the axes through an angle $\varphi$ to eliminate the $x y$ term. This corresponds to a change of co-ordinates given by the equations $x=x^{\prime} \cos \varphi+y^{\prime} \sin \varphi$, 
$y=-x^{\prime} \sin \varphi+y^{\prime} \cos \varphi$. The condition that the coefficient of $x^{\prime} y^{\prime}$ is 0 leads to the equation $(a-b) \sin (2 \varphi)+2 h \cos (2 \varphi)=0$.

Let $s=\sqrt{(a-b)^{2}+4 h^{2}}$. If $s=0$, then any $\varphi$ will work, otherwise there exists $\varphi$ satisfying $\cos (2 \varphi)=(b-a) / s$ and $\sin (2 \varphi)=2 h / s$.

After this change of co-ordinates the equation of $K$ is of the form $A x^{\prime 2}+B y^{2}+2 G x^{\prime}+$ $2 F y^{\prime}+d=0$, where $A=(a+b-s) / 2, B=(a+b+s) / 2$.

If $A=0$ and $G \neq 0$, then $K$ is a parabola, and after the change of co-ordinates $x^{\prime}=x^{\prime \prime}+\left(F^{2}-B d\right) / 2 B G, y^{\prime}=y^{\prime \prime}-F / B$, we can write the equation of $K$ as $B y^{\prime \prime 2}+2 G x^{\prime \prime}=0$. The other case when $K$ is a parabola is when $B=0$ and $F \neq 0$, then we use the change of co-ordinates $x^{\prime}=x^{\prime \prime}-G / A, y^{\prime}=y^{\prime \prime}+\left(G^{2}-A d\right) / 2 A F$ to write the equation of $K$ as $A x^{\prime \prime 2}+2 F y^{\prime \prime}=0$.

If $K$ is not a parabola, then $A B \neq 0$ or $A=G=0$ or $B=F=0$. In these cases let $u=G / A$ if $A \neq 0$ and $u=0$ if $A=0$, and let $v=F / B$ if $B \neq 0$ and $v=0$ if $B=0$. After the change of co-ordinates $x=x^{\prime \prime}-u, y^{\prime}=y^{\prime \prime}-v$, we can write the equation of $K$ as $A x^{\prime \prime 2}+B y^{\prime \prime 2}+D=0$, where $D=d-u^{2}-v^{2}$.

It is important to note that although $\varphi$ is not unique, it is only determined up to a multiple of $\pi$ in general, and is completely arbitrary if $K$ is a circle, the final form of the equation is unique.

As described in [2], the quantities $I_{1}=a+b, I_{2}=a b-h^{2}$ and

$$
I_{3}=\operatorname{det}\left(\begin{array}{lll}
a & h & g \\
h & b & f \\
g & f & d
\end{array}\right)
$$

are invariant under translation and rotation of the axes. Therefore $I_{1}=A+B, I_{2}=A B$ and if $K$ is not a parabola, then $I_{3}=A B D$.

Key Observation. $K$ is an ellipse with semi-minor axis of length 1 if and only if $0<A \leq B=-D$ or $0>B \geq A=-D$. In either case we have $(A+D)(B+D)=0$, and after multiplying by $(A B)^{\overline{2}}$ and expanding, we obtain

$$
I_{3}^{2}+I_{1} I_{2} I_{3}+I_{2}^{3}=0 .
$$

This equation is also satisfied if $K$ is the union of two parallel lines or a double line, since this is equivalent to $I_{2}=I_{3}=0$.

Our condition is equivalent to the condition in [2], which says that if $K$ is an ellipse, then it has semi-minor axis of length 1 if and only if $\lambda=1$ is the larger root of the quadratic equation $I_{3}^{2} \lambda^{2}+I_{1} I_{2} I_{3} \lambda+I_{2}^{3}=0$.

The left-hand side of (1) is a homogeneous polynomial of degree 6 in $a, b, d, f, g$, $h$, since $I_{k}$ is a homogeneous polynomial of degree $k$ for $k=1,2$ and 3 .

We now consider the centres of the four spheres in the plane. If they are all collinear, then, of course, there are infinitely many lines tangent to all four spheres. In all other cases there are at most two pairs of parallel lines passing through all four centres.

Any four points on an ellipse form a convex quadrilateral, so if the four centres do not form a convex quadrilateral, then there is no ellipse through them and there are at most four lines tangent to all the four spheres. 
From now on we assume that the four centres form a convex quadrilateral. We can then choose co-ordinates such that the intersection of the diagonals is the origin, and the four centres have co-ordinates $c_{1}=\left(u_{1}, 0\right), c_{2}=\left(\alpha u_{2}, u_{2}\right), c_{3}=\left(-u_{3}, 0\right)$ and $c_{4}=\left(-\alpha u_{4},-u_{4}\right)$ with $u_{i}>0$ for $1 \leq i \leq 4$.

Let

$$
Q_{0}(x, y)=\left(u_{2} x+\left(u_{1}-\alpha u_{2}\right) y-u_{1} u_{2}\right)\left(u_{4} x+\left(u_{3}-\alpha u_{4}\right) y+u_{3} u_{4}\right)
$$

be the product of the equations of the lines $c_{1} c_{2}$ and $c_{3} c_{4}$, and let

$$
Q_{1}(x, y)=\left(u_{2} x-\left(u_{3}+\alpha u_{2}\right) y+u_{2} u_{3}\right)\left(u_{4} x-\left(u_{1}+\alpha u_{4}\right) y-u_{1} u_{4}\right)
$$

be the product of the equations of the lines $c_{2} c_{3}$ and $c_{1} c_{4}$. Our choice of scaling ensures that $Q_{0}(0,0)=Q_{1}(0,0)=-u_{1} u_{2} u_{3} u_{4}<0$, which we use later.

Let $Q_{t}(x, y)=Q_{0}(x, y)+t\left(Q_{1}(x, y)-Q_{0}(x, y)\right)$ for $t \in \mathbb{R}$ and $Q_{\infty}(x, y)=$ $Q_{1}(x, y)-Q_{0}(x, y)$. Let

$$
Q_{t}(x, y)=a(t) x^{2}+2 h(t) x y+b(t) y^{2}+g(t) x+f(t) y+d(t)
$$

for $t \in \mathbb{R} \cup\{\infty\}$. Let $K_{t} \subset \mathbb{R}^{2}$ be the conic defined by $Q_{t}(x, y)=0$. Then the real pencil of conics passing through $c_{1}, c_{2}, c_{3}$ and $c_{4}$ is exactly $\left\{K_{t} \mid t \in \mathbb{R} \cup\{\infty\}\right\}$. As $Q_{0}(0,0)=Q_{1}(0,0), K_{\infty}$ is the union of the lines $c_{1} c_{3}$ and $c_{2} c_{4}$.

Let $A(t), B(t), D(t), I_{1}(t), I_{2}(t)$ and $I_{3}(t)$ be the values of $A, B, D, I_{1}, I_{2}$ and $I_{3}$ calculated from $a(t), b(t), d(t), f(t), g(t)$ and $h(t)$. (If $K_{t}$ is a parabola, then $D(t)$ is not defined, but the others always are.)

The coefficients of $Q_{t}$ are polynomials of degree at most 1 in $t$, therefore the left-hand side of (1) is a polynomial of degree at most 6 in $t$, so it has at most six solutions in $t$. $K_{\infty}$ is a union of two lines, not an ellipse, so lines tangent to the four spheres occur at finite values of $t$. This means that there are at most six values of $t \in \mathbb{R} \cup\{\infty\}$ such that $K_{t}$ is an ellipse with semi-minor axis of length 1 , and hence there are at most twelve lines tangent to all four spheres.

The quadratic equation $I_{2}(t)=0$ has two roots, $t_{1}=\left(\sqrt{u_{2} u_{3}}-\sqrt{u_{1} u_{4}}\right)^{2} /\left(\left(u_{1}+\right.\right.$ $\left.\left.u_{3}\right)\left(u_{2}+u_{4}\right)\right)$ and $t_{2}=\left(\sqrt{u_{2} u_{3}}+\sqrt{u_{1} u_{4}}\right)^{2} /\left(\left(u_{1}+u_{3}\right)\left(u_{2}+u_{4}\right)\right)$. It is easy to check that $0 \leq t_{1}<t_{2} \leq 1$.

We can write $I_{2}(t)=\eta\left(t-t_{1}\right)\left(t-t_{2}\right)$, where $\eta=a_{\infty} b_{\infty}-h_{\infty}^{2}$ is the coefficient of $t^{2}$ in $I_{2}(t) . \eta$ is negative, because it is the discriminant of the homogeneous degree 2 component of $Q_{\infty}(x, y)$, and $K_{\infty}$ is the union of two intersecting lines.

Therefore $I_{2}(t)=A(t) B(t)>0$ if and only if $t_{1}<t<t_{2}$, so $K_{t}$ is an ellipse if and only if $t_{1}<t<t_{2}$.

By direct calculation,

$$
A\left(\frac{t_{1}+t_{2}}{2}\right)=\frac{u_{1} u_{3}+\left(1+\alpha^{2}\right) u_{2} u_{4}-\sqrt{\left(u_{1} u_{3}+\left(1+\alpha^{2}\right) u_{2} u_{4}\right)^{2}-4 u_{1} u_{2} u_{3} u_{4}}}{2},
$$

which is clearly positive. Therefore $A(t)>0$ for $t \in\left(t_{1}, t_{2}\right)$. (This is the point where the fact that $Q_{0}(0,0)=Q_{1}(0,0)<0$ is used.)

The graphs of $A(t)$ and $B(t)$ are two branches of a hyperbola, possibly each degenerating into the union of two rays, if the hyperbola degenerates into the union of two 
intersecting lines. As $A(t) \leq B(t)$ for all $t$, the fact that $A(t)>0$ for $t \in\left(t_{1}, t_{2}\right)$ implies that $B(t)>0$ for all $t$ and $A(t)>0$ if and only if $t_{1}<t<t_{2} . A\left(t_{1}\right)=A\left(t_{2}\right)=0$ and $A(t)<0$ if $t<t_{1}$ or $t>t_{2}$.

As $K_{t}$ always has real points, we must have $D(t)<0$ for $t_{1}<t<t_{2}$. Therefore the ellipses we are looking for correspond to solutions of $B(t)+D(t)=0$ with $t_{1}<t<t_{2}$.

We also have $I_{3}(t)=\gamma t(t-1)$, where $\gamma$ is independent of $t$. This can be verified by direct calculation. Another way to see it is that $I_{3}(t)=0$ means that $K_{t}$ is degenerate. This only happens at $t=0, t=1$ and $t=\infty$, this is why we get a quadratic instead of the expected cubic. Furthermore, $\gamma>0$, since if $t_{1}<t<t_{2}$, then $A(t)>0, B(t)>0$ and $D(t)<0$. This inequality can also be derived by calculating the explicit formula for $\gamma$.

We now consider three cases.

Case 1: No two sides of the quadrilateral $c_{1} c_{2} c_{3} c_{4}$ are parallel. This is equivalent to $0<t_{1}<t_{2}<1$. Now $A(0)<0, D(0)=A\left(t_{1}\right)=0$. We have

$$
D(t)=\frac{I_{3}(t)}{I_{2}(t)}=\frac{\gamma t(t-1)}{\eta\left(t-t_{1}\right)\left(t-t_{2}\right)} .
$$

The inequalities $\gamma>0$ and $\eta<0$ imply that $\lim _{t \rightarrow t_{1}-} D(t)=\infty$. Both $A(t)$ and $D(t)$ are continuous on $\left[0, t_{1}\right)$, therefore there exists a $t \in\left(0, t_{1}\right)$ such that $A(t)+D(t)=$ 0 . Similarly, there exists a $t \in\left(t_{2}, 1\right)$ such that $A(t)+D(t)=0$. This means that $B(t)+D(t)=0$ can have at most four solutions, giving at most eight lines.

Case 2: Two sides of quadrilateral $c_{1} c_{2} c_{3} c_{4}$ are parallel, but the other two are not. We may assume that $c_{1} c_{4}$ and $c_{2} c_{3}$ are parallel. This is equivalent to $0<t_{1}<t_{2}=1$. By the same argument as before, there exists $t \in\left(0, t_{1}\right)$ such that $A(t)+D(t)=0$. Now $D(t)=\gamma t /\left(\eta\left(t-t_{1}\right)\right)$, so the graph of $-D(t)$ is a hyperbola, and so is the union of the graphs of $A(t)$ and $B(t)$. Two hyperbolas intersect in at most four points, therefore there are at most three solutions to $B(t)+D(t)=0$. There is one pair of parallel lines passing through the four centres, therefore there are at most eight lines tangent to the four spheres.

Case 3: $c_{1} c_{2} c_{3} c_{4}$ is a parallelogram. In this case $t_{1}=0, t_{2}=1$ and $D(t)=\gamma / \eta$ is a constant. Therefore there are at most two solutions to $B(t)+D(t)=0$, there are two pairs of parallel lines passing through the four centres, so there are at most eight lines tangent to the four spheres.

It is easy to construct examples with eight common tangents. The example already given in [2] is if the four centres form a square with sides of length $e$, where $\sqrt{2}<e<2$. Of course, small perturbations of this configuration will also work.

If we do not require the radii of the spheres to be equal, then it is possible to construct four spheres with more than eight common tangent lines. We give a construction with twelve common tangent lines. Theobald proved in [3] that if the centres of four spheres of not necessarily equal radius in $\mathbb{R}^{3}$ are not coplanar and the number of common tangent lines is finite, then this number is at most 12 . A continuity argument then implies that the bound 12 also holds if the centres are coplanar, so our example attains the maximum. 
Let $l$ be the line with direction vector $(1,1,1)$ passing through the point $\left(\frac{1}{6}, 0,-\frac{1}{6}\right)$. The points $(0,1,0),\left(-\sqrt{3} / 2,-\frac{1}{2}, 0\right)$ and $\left(\sqrt{3} / 2,-\frac{1}{2}, 0\right)$ form a regular triangle. The line $l$ is at distance $r_{1}=\sqrt{26} / 6$ from each one of them, so it is tangent to the spheres of radius $r_{1}$ centred at these three points. $l$ is at distance $r_{2}=\sqrt{2} / 6$ from the origin, so let the fourth sphere be the sphere of radius $r_{2}$ centred at $(0,0,0)$. The line $l$ is tangent to these four spheres. This configuration of spheres has a symmetry group of order 12 , generated by reflections in the $x y$-plane, the $x z$-plane and a rotation through $2 \pi / 3$ about the $z$-axis. The line $l$ is not fixed by any of these symmetries, therefore it has 12 distinct images under the action of the group, each of which is a common tangent line to the four spheres. It can be verified by a direct calculation that there are no other common tangent lines.

A similar example can be constructed with any direction vector not parallel or perpendicular to the $x y$-plane, and such that the angle between its orthogonal projection in the $x y$-plane and the $x$-axis is not an integer multiple of $\pi / 6$.

\section{Acknowledgments}

I am grateful to Frank Sottile who mentioned this problem in his talk at the MEGA2000 conference in Bath and who told me about the idea of the ellipses, and to Thorsten Theobald for providing me with a copy of [2] and [3] and for his helpful comments on the manuscript.

\section{References}

[1] P. K. Agarwal, B. Aronov and M. Sharir, Line transversals of balls and smallest enclosing cylinders in three dimensions. Discrete Comput. Geom. 21 (1999), 373-388.

[2] I. G. Macdonald, J. Pach and T. Theobald. Common tangents to four unit balls in $\mathbb{R}^{3}$. Discrete Comput. Geom. 26 (2001), 1-17.

[3] T. Theobald. An enumerative geometry framework for algorithmic line problems in $\mathbb{R}^{3}$. Preprint, 2000.

Received October 9, 2000, and in revised form April 26, 2001. Online publication October 12, 2001. 